\title{
TESTS OF NIOBIUM CATHODE FOR THE SUPERCONDUCTING RADIO FREQUENCY GUN
}

\author{
Qiang Zhao, Triveni Srinivasan-Rao, BNL, Upton, NY 11973, USA \\ Mike Cole, Advanced Energy Systems, Medford, NY 11763, USA
}

\begin{abstract}
For the superconducting all-niobium photocathode radio frequency gun project, we have studied the surface preparation techniques of the niobium cathode material. The quantum efficiency (QE) of high purity niobium $(R R R=250)$ has been intensively measured at room temperature on a dedicated DC system. After buffer chemical polishing or electrolytic polishing, the initial QE is in the order of $10^{-7}$ range tested by a $266 \mathrm{~nm}$ picosecond laser beam. However, the QE could be improved by more than two orders of magnitude after appropriate in situ laser cleaning. Both $266 \mathrm{~nm}$ ps-YAG and $248 \mathrm{~nm}$ nsexcimer laser beams have been used in the process of cleaning. The thresholds of the laser cleaning intensity were also experimentally determined to keep the morphology of the superconducting cavity surface.
\end{abstract}

\section{INTRODUCTION}

For the development of a continuous wave (CW) high brightness electron source, one of the direct and simple approaches is to use the niobium surface of the superconducting (SC) cavity itself as the photocathode [1]. However, without appropriate treatment, the quantum efficiency $(\mathrm{QE})$ of niobium was found to be too low to be of practical use $[2,3]$ at the laser wavelength of interest (e. g. 266nm). Previous experimental results showed that QE of niobium could reach up to $10^{-4}$ by a special preparation technique of a series of diamond mechanical polishing followed by laser cleaning [1]. Since the laser cleaning technique has been successfully applied to the copper and magnesium cathode $[5,6]$, we systematically investigated the laser cleaning effect on the buffer chemical polished (BCP) or electrolytic polished (EP) niobium surface irradiated by the fourth harmonic of a picosecond $\mathrm{Nd}$ :YAG laser or a nanosecond $\mathrm{KrF}$ excimer laser beam. The cleaning laser energy density should be high enough to effectively remove the contamination on the niobium surface. It, however, could not be beyond the damage threshold. Otherwise the morphology of the surface in the cleaned area will be completely changed, which will increase the field emission and thus degrade the performance of the SC cavity. From experiments, we found the effective laser cleaning energy density is around $0.3 \mathrm{~mJ} / \mathrm{mm}^{2}$ for a $15 \mathrm{ps}, 266 \mathrm{~nm}$ laser beam while about one order higher for a $\sim 20 \mathrm{~ns}, 248 \mathrm{~nm}$ excimer laser.

\section{EXPERIMENTAL APPARATUS AND TECHNIQUES}

The picosecond pulses were produced by a $10-\mathrm{Hz}$ mode-locked Nd:YAG laser (Quantel YG 501 DP). The $1.063 \mu \mathrm{m}$ laser out of oscillator is limited to the $\mathrm{TEM}_{00}$ mode by a 1 -mm-diameter intracavity pinhole. A single laser pulse is selected from the train and amplified by an Nd:YAG laser amplifier through two passes. Two KDP (potassium dihydrogen phosphate) crystals are used one after the other to generate $266 \mathrm{~nm}(\mathrm{~h} v=4.66 \mathrm{eV})$ radiation via harmonic conversion. It can produce about $1 \mathrm{~mJ} /$ pulse ultraviolet (UV) beam with pulse duration of about $15 \mathrm{ps}$. This UV beam was utilized for both laser cleaning and QE measurements on the DC system.

The excimer laser (Lambda-Physik LPX-100) operating with $\mathrm{KrF}$ gas mix is capable of producing a $\sim 20 \mathrm{~ns}$ pulse beam with energy of about $250 \mathrm{~mJ} /$ pulse at wavelength of $248 \mathrm{~nm}(\mathrm{hv}=5.0 \mathrm{eV})$. This laser can run at $10 \mathrm{~Hz}$ repetition rate, and was also used for laser cleaning.

The UV beam from laser system was directed onto the cathode by UV mirrors via various optical elements. A He-Ne reference laser and two irises were used to define the trajectory of the UV beam to repeatedly align the beam on the cathode. A half wave plate and a polarizing cube acted as a variable attenuator to control the laser energy. A pyroelectric detector (Molectron J3-09) was used to measure the UV pulse energy entering the vacuum chamber. The laser beam size on the cathode can be adjusted by the variation of the lens position or the irises' apertures. The spatial profile of the UV beam was determined at the equivalent site of the cathode by using a CCD camera system (Spiricon LBA-100A). Figure 1 is the schematic layout of the beam optics for the QE tests.

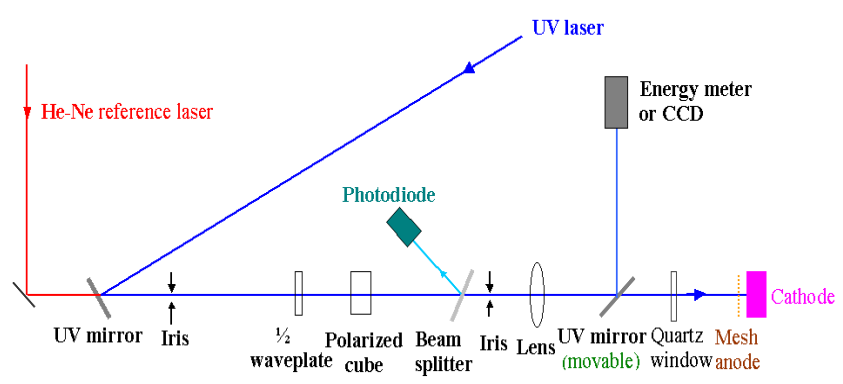

Figure 1: Layout of beam optics for QE test

A 2-3/4 inch stainless steel cubic cell with three fused silica windows was used as the vacuum chamber. The chamber was mounted on an $\mathrm{x}-\mathrm{y}$ translation stage so that it can be remotely moved both horizontally and vertically with a $1 \mu \mathrm{m}$ resolution. This feature was used to scan the cathode for a uniform laser cleaning with the ps laser. A rotary stage with a high voltage and a charge signal feedthrough was placed attached to the cube. The anode and cathode were installed parallel to each other on two aluminum rods attached to the high voltage and the charge signal feed-through, respectively. Thus anode and cathode can be rotated together in vacuum at any angle. 
After the introduction of new samples into the chamber, the system was initially evacuated down to approximately $10^{-6}$ Torr by the successive use of two liquid nitrogen cooled sorption pumps. This pressure was further reduced to around $5 \times 10^{-9}$ Torr after appropriate baking by using a Varian Star-Cell ion pump. The vacuum level in the chamber was monitored by an ionization gauge.

We tried to simulate the SC cavity treatment procedures to prepare the cathode. The niobium cathodes were machined directly from a high purity grade of bulk sample into a cylinder with $9 \mathrm{~mm}$ diameter and $9 \mathrm{~mm}$ thickness. The niobium has the same quality as that used for the SC cavity with residual resistivity ratio (RRR) value of 250 provided by Wahchang Company. All cathodes were then initially cleaned with detergent water. Most of the cathodes were further treated to buffer chemical polishing with the mixture of Fluoric (48\%), Nitic (65\%) and Phosphoric (85\%) acids in a ratio of $1: 1: 2$ by volume. A few samples were cleaned by electropolishing at TJNAL, and some were further mechanically polished after BCP or EP. A compact etching station was established for BCP purpose. The niobium sample was attached on a Teflon holder and immersed into the acids solution with cathode surface facing up. A $50 \mathrm{ml}$ Teflon beaker holding the solution was dipped into an ice-cooled water bath so that the temperature of the solution could be maintained during etching. To facilitate the heat exchange and dislodge any buildup of dissolved material around the sample, the acids mixture was agitated by a magnetic stirrer in the course of BCP etching. The cathodes were typically etched for 15-60 minutes at temperature of 1215 degrees centigrade and then rinsed with deionized (DI) water $(18 \mathrm{M} \Omega)$ and cleaned in an ultrasonic DI water bath. This was followed by high purity of nitrogen drying the sample in a cleaned environment and transport into the vacuum chamber immediately.

In the normal QE measurement, a positive DC high voltage of up to $10 \mathrm{KV}$ was applied to the anode across the diode gap of about $1.5 \mathrm{~mm}$. In order to obtain a uniform field between the anode and cathode, a thin copper mesh (70 lines/inch) was used to cover the anode hole on the cathode side. The charge signal from the cathode was successively amplified by a low noise, charge sensitive pre-amplifier and a shaping main amplifier, and then recorded by a digital oscilloscope (Tektronics 2440) with a $50 \Omega$ termination. The charge measurement system was calibrated by applying a known voltage to a calibrating capacitor in the preamplifier. During QE measurement, photoelectrical charge must be kept low enough to avoid space charge domination and to ensure the charge amplifiers operating at their linear ranges. The laser energy irradiating the cathode was continuously monitored and measured by a fast photodiode calibrated against an energy meter (Molectron J3-09) before and after QE test. To reduce the background from the scattering light in the room, the window of the photodiode was always covered by a UG11 filter. Care was also taken to make sure the photodiode was always working within the linear response range. QE can, therefore, be deduced by simultaneously recording the laser signal from the photodiode together with the charge signal from the cathode on the same scope.

\section{EXPERIMENTAL RESULTS}

When we started to test a newly prepared cathode on the DC system, the initial QE of Niobium was $10^{-7}$ for $\mathrm{BCP}$ and EP samples and $10^{-6}$ for a MP sample. We first used the UV beam from the ps-YAG laser to clean the surface. Tests indicated that QE could be significantly improved when the incident laser energy density was excess of $0.2 \mathrm{~mJ} / \mathrm{mm}^{2}$ for about 50 minutes. Although the QE increased with further increase of energy density, the morphology of cathode surface was totally changed even before QE arrive at its maximum. Such a surface modification would not be acceptable for the SC cavity, since it could induce severe field emission and thus limit the performance of the cavity. Our experiments showed that the optimal energy density was around $0.3 \mathrm{~mJ} / \mathrm{mm}^{2}$ for ps-YAG beam. Figure 2 illustrates the cathode surfaces before and after laser cleaning with different laser energy densities observed under optical microscopes. Some of the samples were also inspected under scanning electron microscopes for higher magnifications.

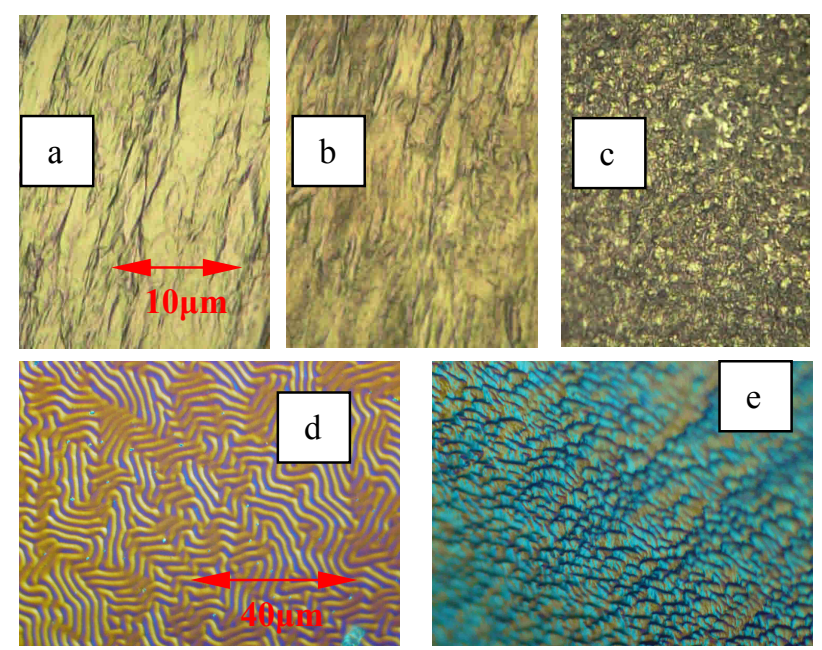

Figure 2: Niobium surfaces cleaned with different psYAG laser energy densities, observed under optical microscope. BCP sample: a) before laser cleaning, b) laser energy density $0.25 \mathrm{~mJ} / \mathrm{mm}^{2}$, c) laser energy density $0.67 \mathrm{~mJ} / \mathrm{mm}^{2}$ EP sample: d) before cleaning; e) laser energy density $0.45 \mathrm{~mJ} / \mathrm{mm}^{2}$

UV beam from excimer laser also demonstrated the capability of cleaning cathode to increase the QE. Since the output energy of excimer laser is more than $200 \mathrm{~mJ}$ and the spatial profile is uniform, it is more efficient to clean a large area without the need of beam scanning. The optimized energy density for this laser was in the region of $3.5 \mathrm{~mJ} / \mathrm{mm}^{2}$.

Since the photoemission of the niobium from the UV laser is a one-photon effect, there was a linear relationship between the energy of incident laser and the charge of the 
emitted electrons as shown in Figure 3. The different intercepts on the log-log graph express different QE. Similar behavior was also observed on cleaning with YAG laser.

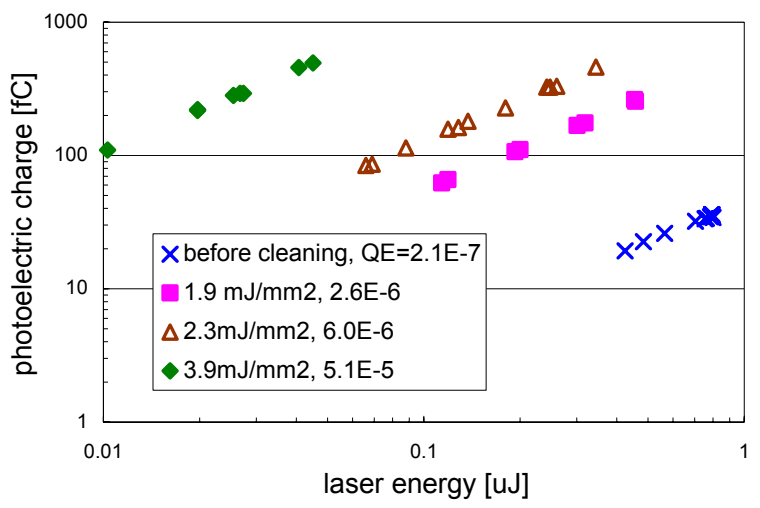

Figure 3: QE of BCP Niobium sample cleaned by $248 \mathrm{~nm}$, measured by $266 \mathrm{~nm}$

Figure 4 is the summary of the typical QE of niobium with different surface treatment. All the measurements were conducted by the $266 \mathrm{~nm}$ YAG laser. BCP, MP+BCP and EP samples were laser cleaned with energy density around $0.3 \mathrm{~mJ} / \mathrm{mm}^{2}$, and $\mathrm{BCP}(\mathrm{HV})$ was cleaned with much lower energy density prior to measurements. Althogh $\mathrm{BCP}(\mathrm{HV})$ cleaning is more effective, extreme care is required since the cathode surface could easily be damaged due to the formation of plasmas. The red bar in $\mathrm{BCP}$ data in Figure 4 corresponds to a heavier BCP cleaning that resulted in doubling of QE.

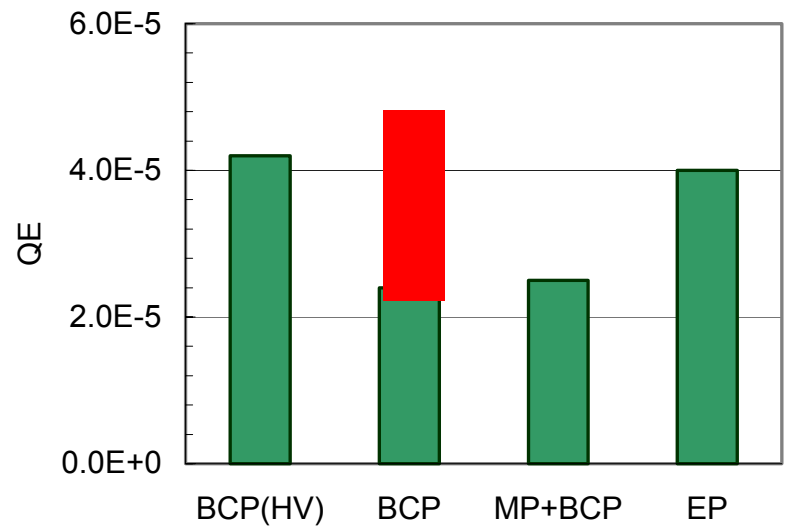

Figure 4: Summary of QE. Laser cleaning energy density was around $0.3 \mathrm{~mJ} / \mathrm{mm}^{2}$ by ps-YAG $(266 \mathrm{~nm})$ for all samples except $\mathrm{BCP}(\mathrm{HV})$. Field on the cathode during measurement was about $1.5 \mathrm{MV} / \mathrm{m}$. BCP (HV): high voltage of $5 \mathrm{KV}$ applied across $1.7 \mathrm{~mm}$ gap during cleaning at $0.1 \mathrm{~mJ} / \mathrm{mm}^{2}$. Red BCP: cathode was heavily BCP cleaned with total remove of $\sim 60 \mathrm{um}$.

Since the gradient on the cathode was lower in the DC system than in the gun cavity, QE was measured as a function of field gradient to take account of the Schottky effect. A typical result is seen in figure 5. By extrapolating this data to $35 \mathrm{MV} / \mathrm{m}$ in the gun cavity, QE of nearly $10^{-4}$ could be achieved.

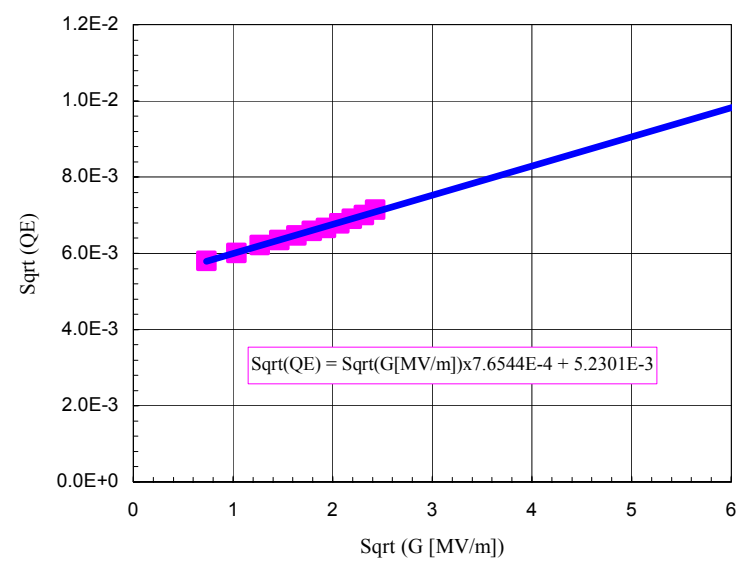

Figure 5: QE vs. field gradient for a BCP sample

\section{SUMMARY}

The QE of BCP and EP Niobium samples increased more than two orders of magnitude after appropriate in situ laser cleaning. Both the UV beams from the ps-YAG and ns-excimer can be used to clean the niobium cathode. The cleaning laser energy densities were determined to avoid the damage of surface. The real QE of niobium in SC cavity at cryogenic temperature will be tested soon.

\section{ACKNOWLEDGMENTS}

The authors would like to thank I. Ben-Zvi for the support and advice. We wish to thank T. Tsang, J. Smedley and R. Geng for the valuable discussions. We acknowledge the expert technical assistance of $\mathrm{M}$. Montemagno and J. Walsh. We also appreciate J. Warren and R. Sabatini for their assistance in performing SEM analysis and JLAB for electro polishing the $\mathrm{Nb}$ samples. This research is supported by the U.S. Department of Energy under Contract No. DE-AC02-98CH10886, DEAC02-98CH10886DOE, and SBIR grant DE-FG0299ER82724.

\section{REFERENCES}

[1] M. Cole, et al., "Development of a Novel SC RF photocathode electron gun", LINAC'2k, p116.

[2] L.N. Hand and U. Happek, "Photoelectric quantum efficiency of niobium for $193 \mathrm{~nm}$ and $248 \mathrm{~nm}$ ", Nucl. Instr. \& Meth. A372 (1996) 335.

[3] A.R. Fry and A.C. Melissinos, "Electron Emission From Metallic Surfaces by Picosecond Laser Pulses", Univ. of Rochester Preprint UR-1340/ER-40685-789, Jan. 1994.

[4] T. Srinivasan-Rao, et al., "Performace of Magnesium Cathode in the S-band RF gun", PAC'97, p2790.

[5] T. Srinivasan-Rao, et al., "Photoemission studies on metals using picosecond UV laser pulses", J. Appl. Phys. 69(1991)3291 